\title{
Functional phosphoproteomic analysis reveals cold-shock domain protein A to be a Bcr-Abl effector-regulating proliferation and transformation in chronic myeloid leukemia
}

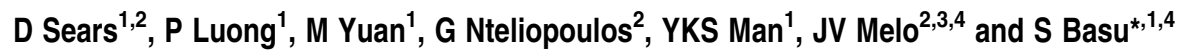

One proposed strategy to suppress the proliferation of imatinib-resistant cells in chronic myeloid leukemia (CML) is to inhibit key proteins downstream of Bcr-Abl. The PI3K/Akt pathway is activated by Bcr-Abl and is specifically required for the growth of CML cells. To identify targets of this pathway, we undertook a proteomic screen and identified several proteins that differentially bind 14-3-3, dependent on Bcr-Abl kinase activity. An siRNA screen of candidates selected by bioinformatics analysis reveals coldshock domain protein A (CSDA), shown previously to regulate cell cycle progression in epithelial cells, to be a positive regulator of proliferation in a CML cell line. We show that Akt can phosphorylate the serine 134 residue of CSDA but, downstream of Bcr-Abl activity, this modification is mediated through the activation of MEK/p90 ribosomal 66 kinase (RSK) signaling. Inhibition of RSK, similarly to treatment with imatinib, blocked proliferation specifically in Bcr-Abl-positive leukemia cell lines, as well as cells from CML patients. Furthermore, these primary CML cells showed an increase in CSDA phosphorylation. Expression of a CSDA phospho-deficient mutant resulted in the decrease of Bcr-Abl-dependent transformation in Rat1 cells. Our results support a model whereby phosphorylation of CSDA downstream of Bcr-Abl enhances proliferation in CML cells to drive leukemogenesis. Cell Death and Disease (2010) 1, e93; doi:10.1038/cddis.2010.72; published online 4 November 2010

Subject Category: Cancer

Chronic myeloid leukemia (CML) is a stem cell disease, in which neoplastic cells carry a translocated Philadelphia chromosome, in which a hybrid $B c r-A B L$ gene encodes a fusion oncoprotein, Bcr-Abl, with constitutive tyrosine kinase activity. Bcr-Abl induces multiple signaling pathways to transduce the oncogenic signal, which ultimately results in uncontrolled proliferation and neoplastic expansion. ${ }^{1} \mathrm{Bcr}-\mathrm{Abl}$ also reduces adhesion of $\mathrm{CML}$ cells to the extracellular matrix and stroma cells, allowing them to bypass the negative influence these interactions have on proliferation. ${ }^{2,3}$ Furthermore, the oncoprotein is believed to inhibit the apoptotic response to mutagenic stimuli, ${ }^{4}$ providing a survival advantage for the leukemic clone, in addition to increasing the likelihood of genomic instability and, therefore, further oncogenic mutations.

Bcr-Abl-dependent pathways include PI3K, MAPK and JAK/STAT, which ultimately control transcription. Targeting the kinase activity of Bcr-Abl via competition with ATP for its binding to the kinase pocket is the basis of the therapeutic action of imatinib mesylate (IM), the preferred drug for front-line treatment of CML. ${ }^{5}$ However, persistence of residual disease or emergence of secondary resistance to $\mathrm{IM}$ is a major cause of concern, especially in the advanced phases of the disease. .,7 $^{6}$

One proposed strategy to suppress the proliferation of IM-resistant cells is to inhibit key proteins downstream of BcrAbl, such as Akt. Previous reports have shown that 14-3-3affinity purification can be used to identify novel Akt substrates in cells in which Akt is activated through exposure to epidermal growth factor. ${ }^{8}$ Owing to the fact that the PI3K/ Akt pathway plays a crucial role in the leukemogenesis of $\mathrm{CML}^{9,10}$ and 14-3-3 binds to a number of well-characterized Akt targets in cancer signaling, ${ }^{11,12}$ we postulated that such 14-3-3 binding would significantly contribute to Bcr-Ablmediated leukemogenesis. We therefore utilized 14-3-3 affinity binding methodology to identify proteins that are regulated by Akt downstream of Bcr-Abl. Here we report that cold-shock domain protein A (CSDA) is a target of Bcr-Ablinduced phosphorylation, regulates proliferation and is critical for Bcr-Abl-induced transformation.

\footnotetext{
${ }^{1}$ Cell Survival Signalling Laboratory, Centre for Molecular Oncology and Imaging, Institute of Cancer, Barts and the London School of Medicine, Queen Mary University of London, London, UK; ${ }^{2}$ Department of Haematology, Imperial College London, London, UK and ${ }^{3}$ Department of Haematology, Centre for Cancer Biology, SA Pathology, Adelaide, South Australia, Australia

*Correspondence: Dr S Basu, Centre for Molecular Oncology and Imaging, Institute of Cancer, Barts and The London School of Medicine and Dentistry, John Vane Science Centre, Charterhouse Square, London EC1M 6BQ, UK. Tel: + 44207882 3839; Fax: + 44207882 3884; E-mail: sub_bas@yahoo.co.uk

${ }^{4}$ These authors contributed equally to this work.

Keywords: CML; Bcr-Abl; CSDA; phosphorylation; proteomics; proliferation; mass spectrometry

Abbreviations: CML, chronic myeloid leukemia; IM, imatinib mesylate; ATIC, 5-aminoimidazol-4-carboxamide ribonucleotide formyltransferase/IMP cyclohydrolase; GPI, glucose phosphate isomerase; CSDA, cold-shock domain protein A; RSK, p90 ribosomal S6 kinase

Received 03.8.10; revised 28.9.10; accepted 29.9.10; Edited by G Melino
} 


\section{Results}

Identification and comparative analysis of IM-sensitive 14-3-3 binding proteins in Bcr-Abl-positive CML cells. To identify Bcr-Abl-dependent 14-3-3 binding proteins, GST-14-3-3-affinity purification was utilized in combination with mass spectrometry to identify sufficient numbers of 14-3-3 bound proteins to detect differential binding after inhibition of Bcr-Abl kinase activity. The affinity purification was undertaken in whole-cell lysates from LAMA84 CML cells cultured in the presence or absence of IM as described in Materials and Methods (Figure 1a). Phosphotyrosine western blots of lysate inputs for the affinity purification displays a significant reduction in the level of tyrosine phosphorylation of multiple proteins after treatment with IM, confirming efficacy of the inhibitor in our assay (Figure 1b). Furthermore, enrichment of tyrosinephosphorylated proteins was detected among the untreated 14-3-3 binding proteins in the pulldown (Figure 1b).

In total, 318 proteins were identified by mass spectrometry as being bound to 14-3-3 (Supplementary Table 1). In order to assess the robustness of the GST-14-3-3-affinity purification technique, we performed bioinformatic analysis of published interactions (www.hrpd.org) and confirmed that a number of proteins that bound to 14-3-3 in our affinity purification screen had previously been shown to bind 14-3-3 (Supplementary Table 2). The previous identification of these proteins as 14-3-3 binding proteins was achieved through a variety of techniques, including GST-affinity purification, Tandemaffinity purification and immunoprecipitation. ${ }^{13-22}$ This reassured us that the eluates from the GST-14-3-3-affinity purifications were highly enriched for 14-3-3 binding proteins and that the proteins we identified were not artifactual results generated by the use of the GST-14-3-3-affinity purification technique.

Of the 318 proteins, 69 bound 14-3-3 only in the absence of IM treatment. We focused on these proteins since we were interested only in putative Akt targets that were regulated by Bcr-Abl activity in CML. In accord with other 14-3-3-affinity purification screens and the critical role of PI3K/Akt signaling in cancer cell biology, ${ }^{15,17,23}$ grouping of these proteins by biological function, as described by the Human Protein a

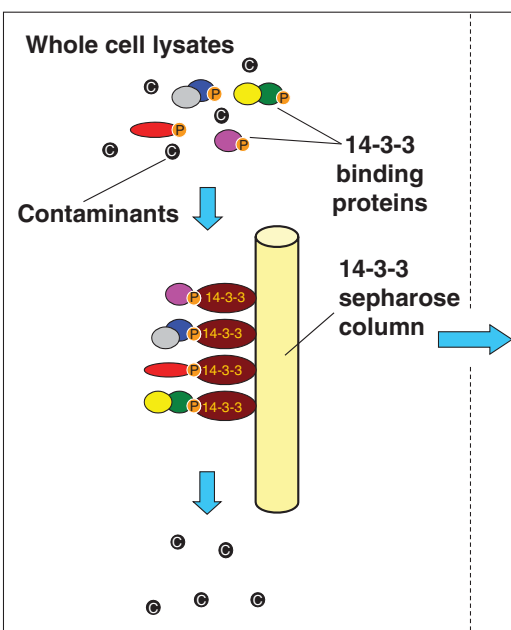

b

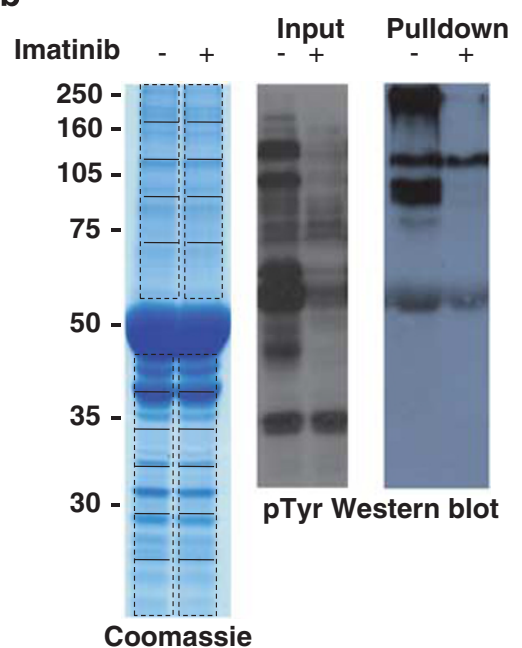

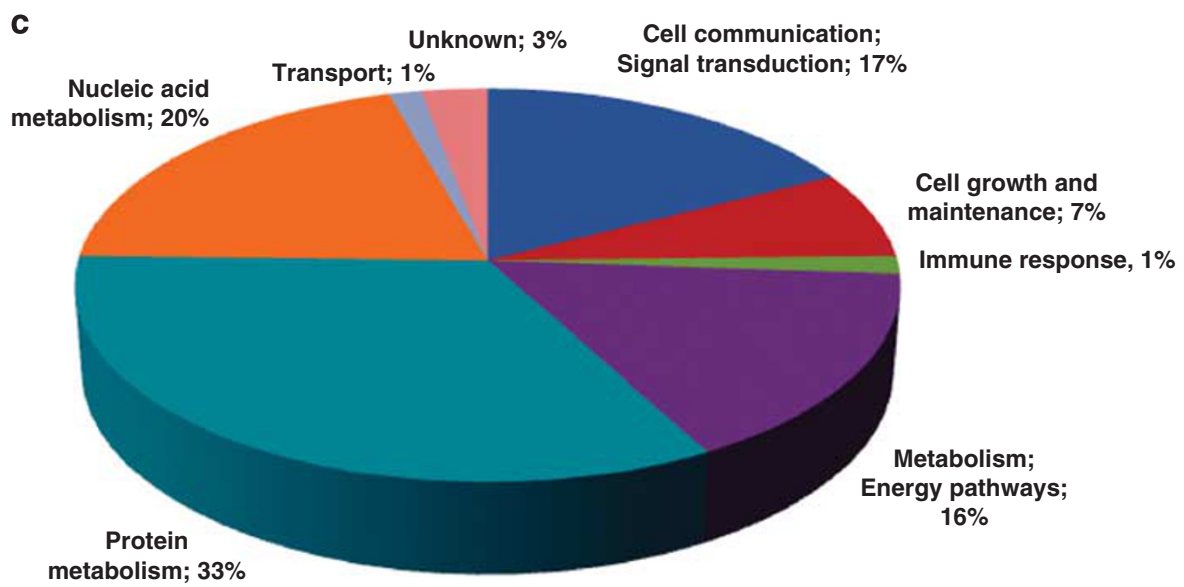

Figure 1 Identification and analysis of Bcr-Abl-dependent 14-3-3-interacting proteins. (a) Schema of GST-14-3-3-affinity purification from IM-sensitive CML cells. Lysates from LAMA84 cells untreated or treated with $5 \mu \mathrm{M}$ for $2 \mathrm{~h}$ were incubated with GST-14-3-3-sepharose; bound proteins were eluted and identified by mass spectrometry as described in Materials and Methods. (b) Aliquots of eluates from affinity purification were analyzed by Coomassie staining and western blot with phospho-tyrosine antibody. (c) Proteins that bound 14-3-3 only in the absence of IM were grouped by biological functions as described in the text 
Reference Database (www.hrpd.org), enriched for protein metabolism (33\%), nucleic acid metabolism (20\%), signal transduction (17\%), as well as energy and metabolism (16\%) (Supplementary Table 3, Figure 1c).

siRNA screen for proliferation of candidate Bcr-Abldependent Akt targets. On the basis of phosphorylationsite motif analysis for 14-3-3 binding and Akt kinase motifs (http://scansite.mit.edu), as well as functional analysis, 20 of the 69 proteins that bound to 14-3-3 only in cells with active Bcr-Abl were selected for inclusion in a customized siRNA screen for proliferation (Supplementary Table 4). Two siRNA sequences were used for any gene that had pre-validated siRNAs, whereas three siRNA sequences were used for genes for which two pre-validated siRNAs were not available (Supplementary Table 5). The K562 cell line was used for the siRNA screen because of their relatively high transfection efficiency as well as their wide use in studying CML. Cells were transfected with the siRNA oligonucleotides and proliferation assessed by MTS $48 \mathrm{~h}$ post transfection as described in Materials and Methods. Proliferation was normalized to a non-targeting siRNA control and a 'cell death' siRNA was included as a positive control for siRNAmediated decrease of proliferation (Figure 2a).

As shown in Figure 2a, transfection of three of the 20 candidates included in the screen resulted in $>20 \%$ decrease of proliferation: 5-aminoimidazol-4-carboxamide ribonucleotide formyltransferase/IMP cyclohydrolase (ATIC, Entrez Gene ID 471), glucose phosphate isomerase (GPI, Entrez Gene ID 2821) and CSDA (Entrez Gene ID 8531). The latter has been shown to be a transcription factor, shuttling between tight junctions and nuclei, promoting cell proliferation. ${ }^{24,25}$ We selected CSDA (also called ZONAB) for further analysis as besides enhancing cell proliferation, it has recently been implicated in breast cancer metastasis and shown to be upregulated in gastric cancer. ${ }^{26,27}$ Its role in CML or other
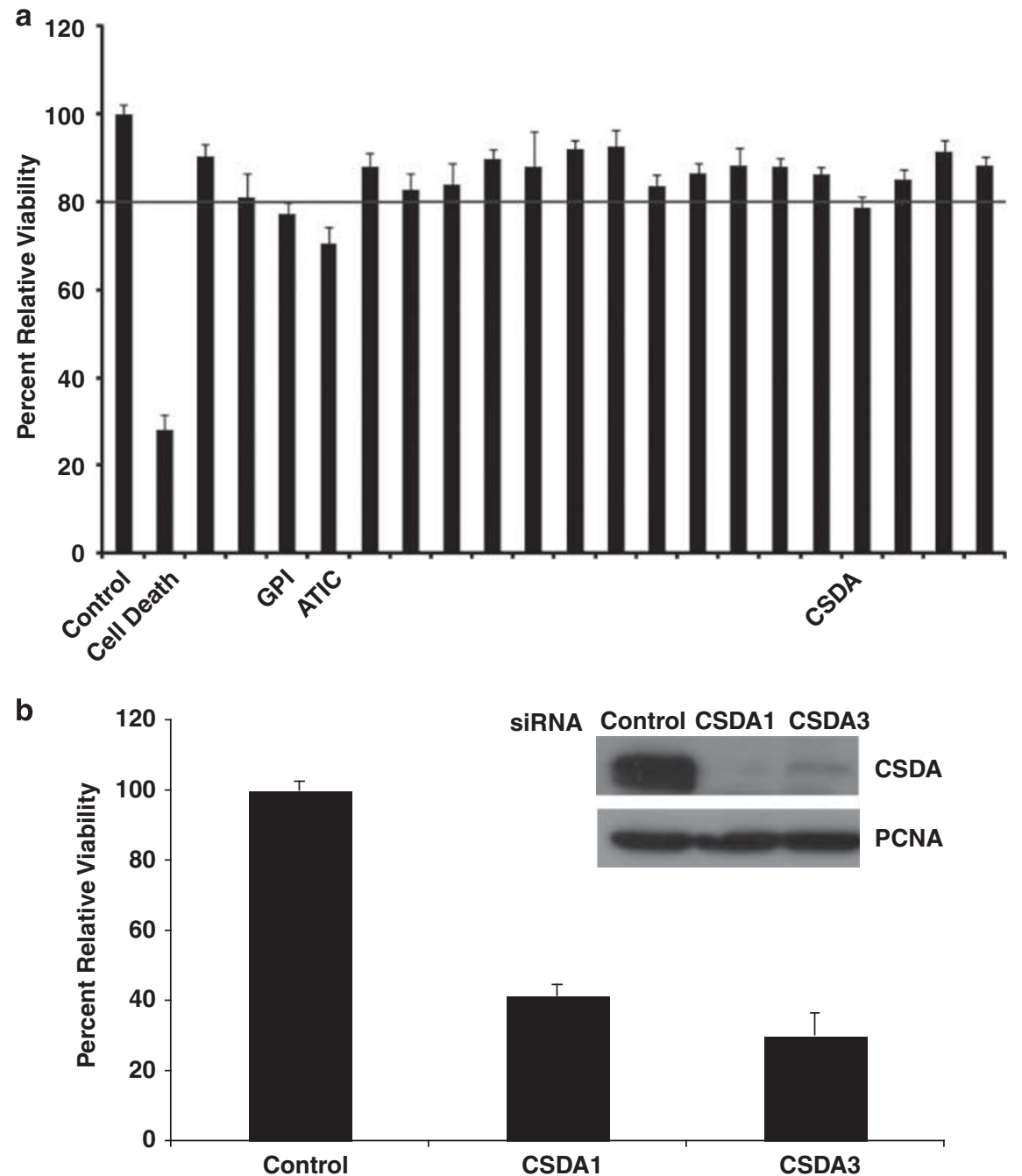

Figure 2 Targeted siRNA screen of selected candidate Akt effectors reveal CSDA to regulate proliferation in CML. (a) Twenty IM-sensitive 14-3-3 interactors were selected based on biological function and presence of putative Akt phosphorylation motif for inclusion in the siRNA screen. Two or three targeting siRNA sequences to each candidate were transfected in K562 cells for $48 \mathrm{~h}$ and averaged for readings of proliferation by MTS as described in Methods and Methods. The three candidates whose silencing decreased proliferation greater than 20\% are indicated. (b) Transfection of CSDA siRNA targeting sequences 1 and 3 effectively silenced protein expression on K562 cells and greatly decreased proliferation after $72 \mathrm{~h}$ 
hematological malignancies, however, has not been in investigated. Two of the three siRNAs to CSDA could effectively silence endogenous protein expression in K562 cells as shown by western blot and transfection with these individual siRNAs for $72 \mathrm{~h}$ exhibited an even more profound effect on cell proliferation (Figure $2 b$ ). We further analyzed CSDA silenced K562 cells by Annexin V/propidium iodide (PI) staining as well as cell cycle analysis by $\mathrm{PI}$ and determined that CSDA expression regulates proliferation and not apoptosis (Supplementary Figure 1A) or cell cycle arrest (Supplementary Figure 1B).

CSDA is phosphorylated in a Bcr-Abl-dependent manner in CML cells and CML patient samples. Examining the CSDA amino-acid sequence, we noted striking similarity with the recently identified Akt site on YB-1, ${ }^{28}$ a closely related cold-shock domain containing protein. We generated a phospho-deficient serine to alanine construct (CSDAS134A) targeting the putative Akt site and expressed it along with wild-type CSDA in 293 cells. Western blot analysis with a phospho-specific commercial antibody (pYB1) to the homologous site on YB-1 (serine 102) determined that this reagent could be used to detect specific phosphorylation on serine S134 of CSDA (Figure 3a).

We next developed FLAG-CSDA-expressing stable LAMA84 and K562 cell lines to assess Bcr-Abl-dependent phosphorylation of CSDA in CML. As shown in Figure 3b, immunoprecipitation of FLAG-CSDA followed by immunoblotting with pYB-1 of lysates from both stable cell lines treated with IM results in profound decrease in $\mathbf{S 1 3 4}$ phosphorylation of CSDA, revealing Bcr-Abl-dependent phosphorylation.

To determine whether Bcr-Abl-dependent CSDA phosphorylation is detectable in actual CML, we purified CD34 + cells from leukaphareses from CML patients or normal donors as described in Materials and methods. Western blot analysis (Figure 3c) revealed that lysates derived from $\mathrm{CML}$ cells (sample ID 37 and 44) show Bcr-Abl expression and a concomitant increase in phosphorylation of CrkL, a substrate for Bcr-Abl and a widely used readout for Bcr-Abl activity $^{29,30}$ relative to lysates from healthy individuals (sample ID 35 and 43). Tellingly, CML cells also exhibited increased phosphorylated CSDA signal relative to CSDA expression as compared with normal cells, indicating greater specific phosphorylation of CSDA at serine 134 in Bcr-Abl-positive CML.

Bcr-Abl-induced CSDA phosphorylation is independent of Akt, but dependent on MEK/RSK pathway. Bcr-Abl activates a number of downstream signaling pathways besides PI3K/Akt. To investigate whether Bcr-Abl-induced CSDA phosphorylation is dependent on Akt activity, we co-expressed CSDA with either empty vector or Bcr-Abl in 293 cells, and treated them with control vehicle or a specific Akt inhibitor (Akti VIII). Western blot analysis revealed that Akt inhibition is effective in decreasing the basal CSDA phosphorylation in vector control cells, but not in Bcr-Abltransfected cells (Figure 4a).

In order to determine which kinases are responsible for Bcr-Abl-induced CSDA phosphorylation, we co-transfected

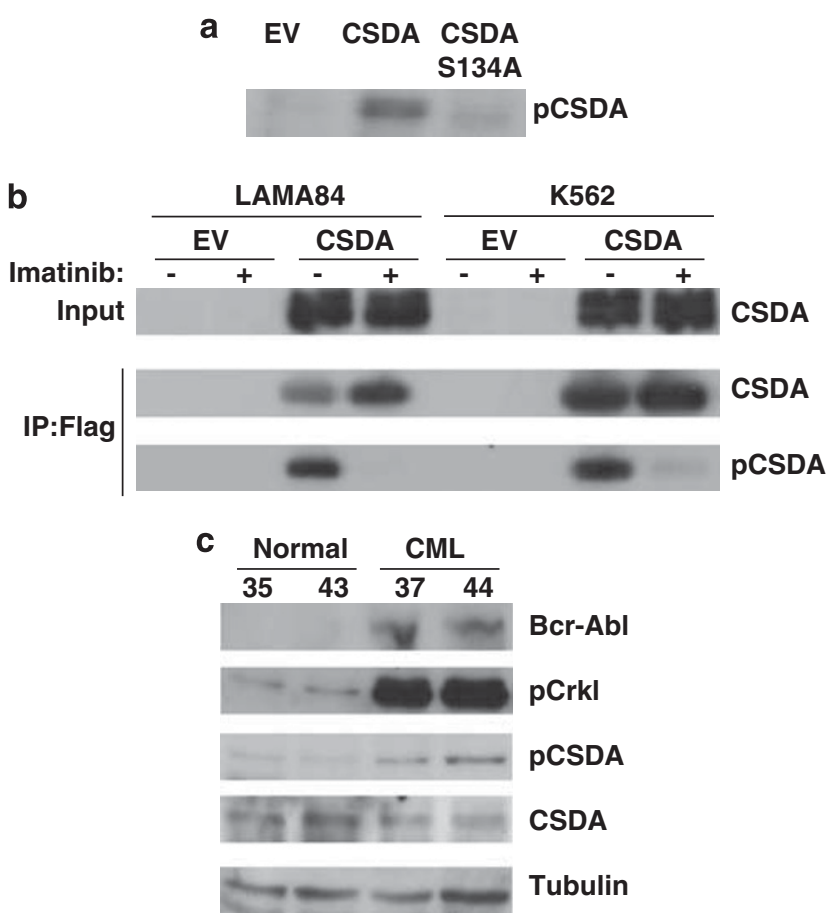

Figure 3 CSDA is phosphorylated at serine 134 in a Bcr-Abl-dependent manner in CML cell lines and CD34 + -purified primary CML cells. (a) The 293 cells were transfected with empty vector, pCMV2B-CSDA or pCMV2B-CSDAS134A for $48 \mathrm{~h}$ and lysates analyzed by western blot with pYB-1 antibody to detect CSDA phosphorylation as described in the text. (b) LAMA84 and K562 cells were stably transfected with empty vector or PBABE_flagCSDA and treated or not (DMSO) with $1 \mu \mathrm{M}$ IM for $2 \mathrm{~h}$. Lysates were immunoprecipitated with FLAG M2 beads and inputs and eluates were analyzed by western blot for the expression of phosphorylated CSDA and of total CSDA by anti-ZONAB. (c) CD34+-purified cells from leukaphareses from normal individuals (sample ID 35,43 ) or CML patients (sample ID 37, 44) were lysed and analyzed by western blot for the expression of Bcr-Abl by anti-c-Abl, phosphorylated CrkL, phosphorylated CSDA, CSDA and tubulin

293 cells with CSDA and empty vector or Bcr-Abl, and treated them with control vehicle, mTOR inhibitor (rapamycin), PI3K inhibitor (LY294002), active MEK inhibitor (U0126), MEK inhibitor (PD98059) or p38 MAPK inhibitor (SB203580). As shown in the top panel of Figure $4 \mathrm{~b}$, treatment with the PI3K inhibitor but not the other inhibitors reduced basal CSDA phosphorylation. Similar to our findings with Akt (Figure 4a), PI3K inhibition did not affect Bcr-Abl-induced CSDA phosphorylation (Figure 4b, bottom panel). Strikingly, both MEK inhibitors were ineffective in reducing basal phosphorylation (Figure 4b, top panel), but robustly reduced Bcr-Abl-induced CSDA phosphorylation.

Sequence analysis led us to expect that MEK does not directly phosphorylate CSDA at serine 134. However, the p90 ribosomal S6 kinase (RSK) was recently reported to phosphorylate the conserved site on YB-1 and RSK signaling downstream of MEK activity is well established. ${ }^{31,32}$ Tellingly, treatment with an RSK inhibitor (SL0101) blocked Bcr-Ablinduced CSDA phosphorylation as effectively as MEK inhibition (Figure 4c). These data suggest that although Akt phosphorylates CSDA at serine 134 in non-CML cells, Bcr-Abl activity results in MEK-dependent RSK phosphorylation of CSDA at the same site. 
RSK inhibition specifically blocks proliferation in Bcr-Abl-positive cell lines and primary CML cells. We investigated whether RSK activity is selectively critical in Bcr-Abl-positive cells by undertaking a proliferation assay
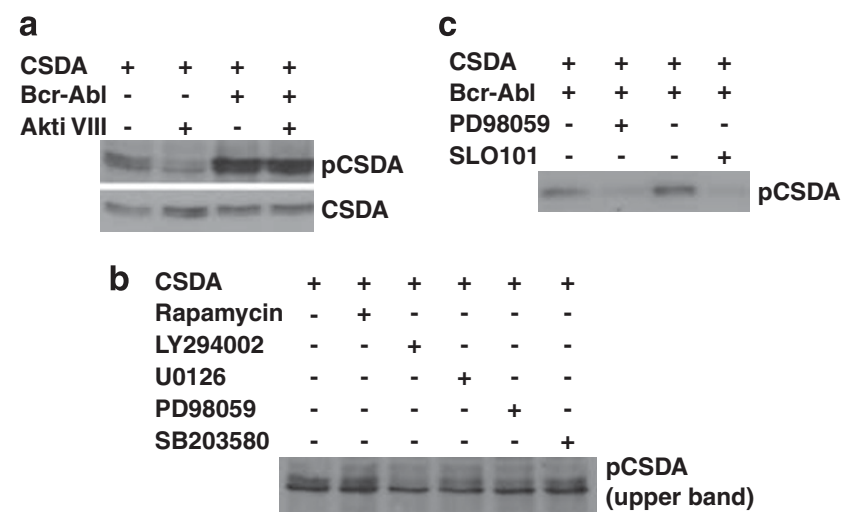

\begin{tabular}{|c|c|c|c|c|c|c|c|}
\hline CSDA & + & + & + & + & + & + & \\
\hline Bcr-Abl & + & + & + & + & + & + & \\
\hline Rapamycin & - & + & - & - & - & - & \\
\hline LY294002 & - & - & + & - & - & - & \\
\hline U0126 & - & - & - & + & - & - & \\
\hline PD98059 & - & - & - & - & + & - & \\
\hline \multirow[t]{2}{*}{ SB203580 } & - & - & - & - & - & + & \\
\hline & & & - & & & - & $\begin{array}{l}\text { pCSDA } \\
\text { (upper band) }\end{array}$ \\
\hline
\end{tabular}

Figure 4 CSDA phosphorylation is mediated by Akt in the absence of Bcr-Abl, but by MEK/RSK signaling downstream of Bcr-Abl. (a) The 293 cells were co-transfected with pCMV2B-CSDA and empty vector or pCDNA3.1Bcr-Abl as indicated for $48 \mathrm{~h}$. Cells were then serum-starved overnight, treated or not (DMSO control) with $10 \mu \mathrm{M}$ Akti VIII for $2 \mathrm{~h}$, and then serum was reintroduced (to 10\%) for $30 \mathrm{~min}$. Lysates were analyzed by western blot for CSDA and phosphorylated CSDA expression as described previously. (b) The 293 cells were co-transfected with pCMV2B-CSDA and empty vector (top panel) or pCDNA3.1Bcr-Abl (bottom panel), serum starved as in (a), and treated or not (DMSO control) with the inhibitors rapamycin $(80 \mathrm{nM}), \mathrm{LY} 294002(50 \mu \mathrm{M}), \mathrm{U} 0126(20 \mu \mathrm{M})$, PD98059 $(100 \mu \mathrm{M})$ or SB203580 $(10 \mu \mathrm{M})$ for $2 \mathrm{~h}$, after which $10 \%$ serum was added for $30 \mathrm{~min}$. Lysates were analyzed by western blot for CSDA and phosphorylated CSDA expression as described previously. (c) The 293 cells were co-transfected with pCMV2B-CSDA and pCDNA3.1Bcr-Abl and treated with PD98059 $(100 \mu \mathrm{M})$ or SL0101 $(20 \mu \mathrm{M})$ and lysates analyzed for CSDA and phosphorylated CSDA expression as before comparing K562 and Ramos cell lines. As expected, treatment with IM selectively blocked proliferation in K562 CML cells while having negligible effect on the Bcr-Ablnegative, Ramos Burkitt's lymphoma line, whereas Akt inhibition decreased proliferation in both cell lines (Figure 5a). Strikingly, similar to IM, RSK inhibition reduced proliferation only in the K562 cells (Figure 5a). We assessed whether the difference in sensitivity to RSK inhibitor may be a function of differential S6 kinase activity in these cells. Indeed, although Ramos and K562 cells express similar levels of both RSK1 and RSK2, the K562 CML lines exhibit markedly increased S6 kinase activity as detected by an antibody specific to S6 kinase phosphorylated at Thr389 (Figure 5b).

To determine whether specificity to RSK inhibition is also evidenced in primary CML cells, we compared the proliferation rate of normal and CML CD34 + progenitors treated with IM, Akt inhibitor or RSK inhibitor. Similar to what we observed in the cell lines (Figure 5a), IM-induced Bcr-Abl inhibition and RSK inhibition affected growth only of CML progenitor cells, whereas Akt inhibition abrogated proliferation in both CML and normal cells (Figure $5 \mathrm{c}$ ). These data indicate that inhibition of RSK specifically reduces proliferation in Bcr-Abl-positive cells, both in cell lines and primary CML.

CSDA expression and $\mathbf{S 1 3 4}$ phosphorylation regulates Bcr-Abl-dependent transformation. We have shown that CSDA expression and RSK activity are both critical for proliferation in CML (Figures $2 \mathrm{~b}$ and 5 ). We have also discovered that CSDA is phosphorylated at serine 134 downstream of Bcr-Abl in an RSK-dependent manner (Figure 4). To determine whether CSDA S134 phosphorylation is critical for Bcr-Abl-dependent transformation, we generated stable lines expressing empty vector or coexpressing Bcr-Abl and empty vector, CSDA or the CSDAS134A phospho-deficient mutant in Rat1 cells to employ in soft agar colony formation assays. ${ }^{33,34}$

After selection, we validated the expression of Bcr-Abl and CSDA constructs (Figure 6a). Furthermore, using phosphospecific antibodies to CrkL and CSDA, we confirmed that
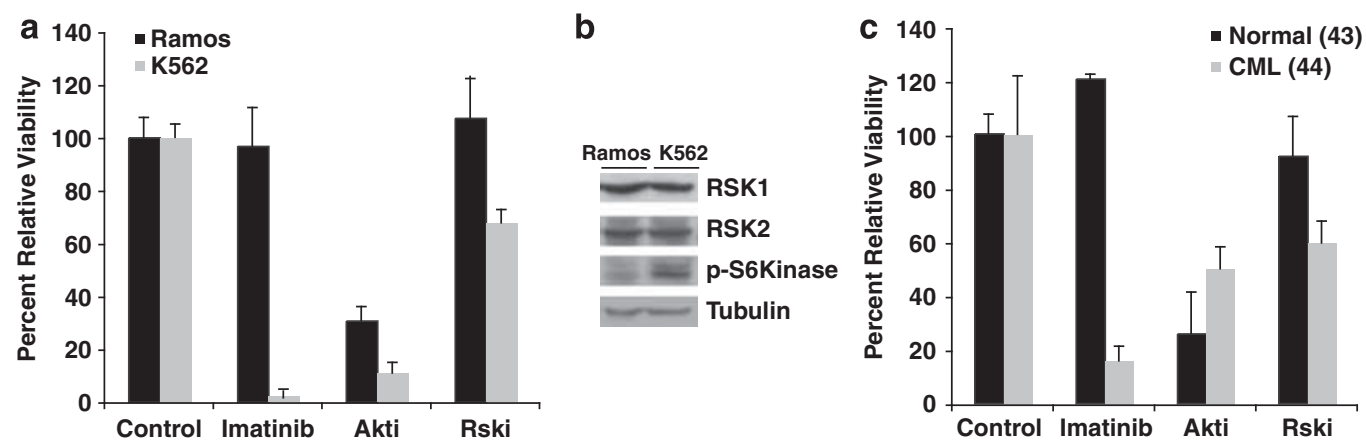

Figure 5 RSK inhibition selectively blocks proliferation in CML cell lines and primary CML cells. (a) Ramos or K562 cells were seeded and treated with DMSO control, IM $(2 \mu \mathrm{M})$ ), Akt inhibitor (10 $\mu \mathrm{M}$ Akti VIII) or RSK inhibitor (20 $\mu \mathrm{M}$ SL0101) as described in Materials and methods. Proliferation was assessed by MTS after $72 \mathrm{~h}$ treatment. (b) Ramos or K562 cell lysates were analyzed by western blot for RSK1, RSK2, phospho-S6 kinase and tubulin as indicated. (c) CD34+-purified cells from leukapharesis from a normal individual (sample ID 43) or a CML patient (sample ID 44) were cultured for $16 \mathrm{~h}$ followed by treatment with DMSO control or indicated inhibitors as in (a). Proliferation was assessed by MTS after $72 \mathrm{~h}$ treatment. Three independent experiments were each carried out in triplicate and data presented are mean value \pm SD of one representative experiment 


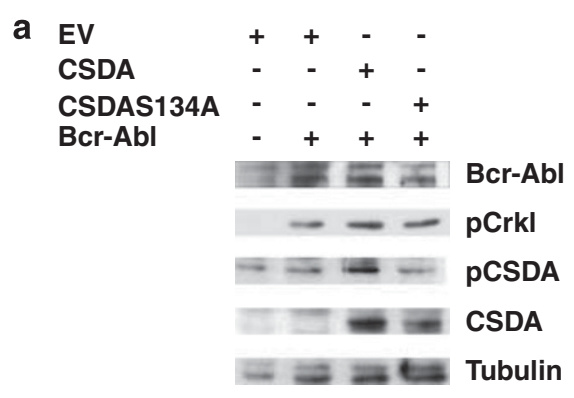

b

EV

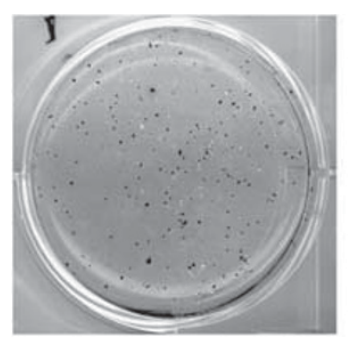

+ Bcr-Abl

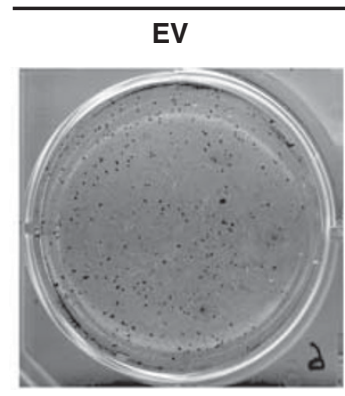

CSDA

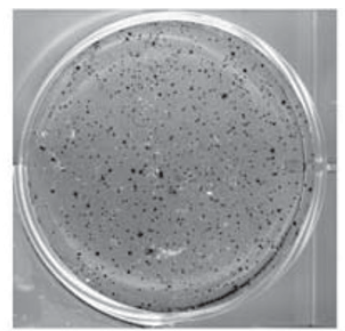

$\star * \star$

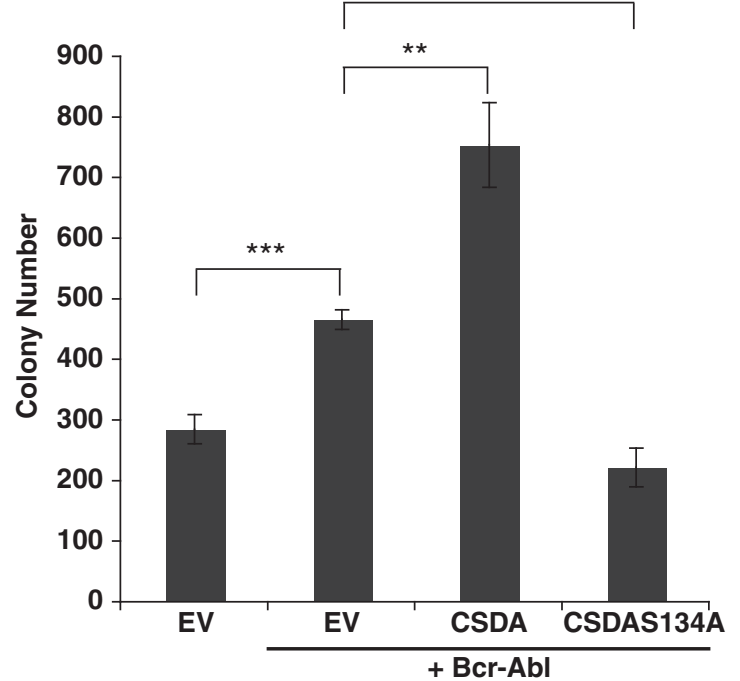

CSDAS134A

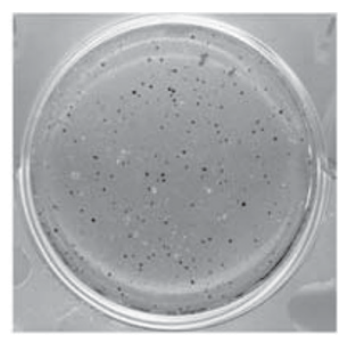

Figure 6 CSDA expression and phosphorylation at serine 134 regulates Bcr-Abl-dependent transformation. (a) Rat1 fibroblasts were stably co-transfected with relevant empty vectors (EV) or pCDNA3.1Bcr-Abl and empty vector or pBabe_flagCSDA or pBabe_flagCSDA as indicated, and lysates analyzed by western blot for the expression of Bcr-Abl, phosphorylated CrkL, phosphorylated CSDA, CSDA and tubulin as described previously. (b) Indicated Rat1 stable cell lines were cultured in soft agar and analyzed for colony formation as described in Materials and methods. Three independent experiments were each carried out in triplicate and data presented are mean value \pm SD of one representative experiment. EV versus $\mathrm{EV}+\mathrm{Bcr}-\mathrm{Abl}:{ }^{* *} t=10.75, P=0.0017$; Bcr-Abl $+\mathrm{EV}$ versus $\mathrm{Bcr}-\mathrm{Abl}+\mathrm{CSDA}:{ }^{*} t=6.971, P=0.02 ; \mathrm{Bcr}-\mathrm{Abl}+\mathrm{EV}$ versus $\mathrm{Bcr}-\mathrm{Abl}+\mathrm{CSDAS134A:}{ }^{* \star *} t=11.9, P=0.0003$. Representative pictures are provided

expressed Bcr-Abl was active, and that expressed CSDA, but not CSDAS134A protein, exhibits increased phosphorylation at serine 134A with Bcr-Abl co-expression (Figure 6a). As shown in Figure $6 \mathrm{~b}$, cells stably expressing Bcr-Abl generated significantly greater number of colonies when cultured in soft agar than cells only expressing empty vector. Colony formation was further and significantly enhanced from cells co-expressing Bcr-Abl and CSDA, but not the phosphodeficient CSDAS134A construct compared with Bcr-Abl and empty vector (Figure $6 \mathrm{~b}$ ). In fact, co-expression of Bcr-Abl and CSDAS134A resulted in lower number of colonies than Bcr-
Abl alone. These findings reveal that CSDA phosphorylation as well as expression is critical for Bcr-Abl-mediated malignant transformation.

\section{Discussion}

We showed that CSDA is specifically phosphorylated at serine 134 downstream of Bcr-Abl in CML cell lines and primary progenitor cells from CML patients (Figure 3 ). However, we unexpectedly determined that CSDAS134 phosphorylation is dependent on Akt in the absence of 
Bcr-Abl, but dependent on MEK/RSK downstream of Bcr-Abl (Figure 4). RSK targets such as S6 are key regulators of translation and S6 phosphorylation downstream of MEK activity was shown to be via RSK rather than mTOR to stimulate cap-dependant translation. ${ }^{35,36}$ Although CSDA has been reported to function as a transcription factor, activating target genes such as PCNA and cyclin D1 that regulate proliferation and morphogenesis in epithelial cells, ${ }^{24,37}$ we did not detect significant changes in specific luciferase activity of either PCNA- or cyclin D1-luciferase constructs, co-expressed with Bcr-Abl and either CSDA or CSDAS134A constructs (data not shown).

It may be that Bcr-Abl-MEK-RSK signaling induces CSDA to regulate translation rather than transcription to promote proliferation in CML. Interestingly, the closely related coldshock domain-containing protein, YB-1, has been shown to regulate translation in breast cancer progression by repressing cap-dependent and promoting cap-independent mechanisms, with Akt phosphorylation at serine 102 resulting in decreased cap-complex binding of YB-1, and thus decreased translational repression in breast and other epithelial cells. ${ }^{28,38-40}$ It will be interesting to determine if there are similar cap-dependent and -independent differences in RSK versus Akt phosphorylation of CSDA at serine 134 (homologous to serine 102 in YB-1) in regulating translation in CML- and Bcr-Abl-negative leukemias, and if blocking CSDAS134 phosphorylation can be exploited to specifically target CML.

We also showed that inhibition of RSK activity specifically blocks proliferation, similarly to CSDA silencing, in an established Bcr-Abl-positive cell line as well as in primary progenitor cells from CML patients (Figure 5). Furthermore, we showed that CSDA, but not the RSK phospho-site mutant, enhances Bcr-Abl-dependent transformation. In fact, co-expression of CSDAS134A blocks Bcr-Abl-dependent transformation. However, it is probable that other RSK phosphorylation targets, both known and undiscovered, are also critical for tumorigenesis, and future work will determine if particular substrates are relevant for specific cancers. Unsurprisingly, proteins involved in protein synthesis, processing and fate were enriched in our bioinformatics analysis of 14-3-3 binding proteins in our initial proteomic screen for BcrAbl effectors in CML (Figure 1c).

In this study, we selected CSDA for further investigation based on its previous known function in regulating cell cycle progression and upregulated expression in epithelial cancers, ${ }^{24,26,27}$ as well as its scoring highly in a secondary targeted siRNA screen for proliferation in K562 CML cells (Figures 2a). Gene ontology analysis of the Bcr-Abl-dependent 14-3-3 binders revealed enrichment for proteins involved in energy and metabolism (Supplementary Table 3, Figure 1c). Interestingly, the other two proteins that scored highly in the siRNA proliferation screen, ATIC, which catalyzes the last two steps of de novo purine biosynthesis, and GPI, which catalyzes the reversible isomerization of glucose-6-phosphate and fructose-6-phosphate, are both key metabolic enzymes linked to hematological malignancies ${ }^{41,42}$ (Figure 2a). Therefore, metabolic regulation downstream of Bcr-Abl can also be targeted in CML, as it has been in therapy for other cancers. ${ }^{43,44}$ The findings in this study indicate that
CSDA phosphorylation and RSK signaling in general may offer an alternative to PI3K/Akt inhibition in targeting Bcr-Abldependent leukemogenesis.

\section{Materials and Methods}

Cell culture, transfection and treatment. LAMA84, K562 and Ramos cell lines were cultured in suspension in RPMI 1640 medium (Invitrogen, Paisley, UK), supplemented with $10 \%$ heat-inactivated fetal calf serum (Harlan Sera-Lab Ltd, Loughborough, UK), $5000 \mathrm{IU} / \mathrm{ml}$ penicillin, $5000 \mu \mathrm{m} / \mathrm{ml}$ streptomycin and $200 \mathrm{mM}$ L-glutamine. The 293T and Rat1 cell lines were cultured in Dulbecco's modified Eagle's media (Invitrogen) supplemented as above.

Transfections of cDNA in 293T and Rat1 cells were performed with Effectene (Qiagen, Crawley, UK) according to the manufacturer's instructions. Rat1 stable cell lines were generated with co-selection in G418 and puromycin (Invitrogen). Retroviral infections of LAMA84 and K562 were performed as described previously, ${ }^{45}$ and stable cell lines were generated with selection in puromycin. siRNA transfection in K562 and Ramos cells were performed with Interferin (Polyplus, Illkirch, France) according to the manufacturer's instructions.

IM was kindly provided by Dr. E Buchdunger (Novartis Pharma, Basel, Switzerland). Akt inhibtor VIII, LY294002, rapamycin, U0126, PD98059 and SB203580 were all purchased from Calbiochem (Nottingham, UK). Cells were treated with inhibitors at the concentrations detailed in the figure legends.

Patient sample preparation. Peripheral blood cells were obtained by leukapheresis from newly diagnosed patients with CML or from normal donors. These cells were portions of leukaphereses processed by the Stem Cell Laboratory, Hammersmith Hospital (London, UK), in excess of clinical requirements. Informed consent for the use of cells for research was obtained with approval from the Hammersmith and Queen Charlotte's and Chelsea Research Ethics Committee Institutional Review Board. CD34 ${ }^{+}$cells were labeled using MiniMACS technology according to the manufacturer's instructions (Miltenyi Biotec, Bisley, UK). Cells were resuspended in $350 \mu \mathrm{l}$ of MACS buffer (phosphate-buffered saline (Gibco, Paisley, UK), $0.5 \%$ bovine serum albumin (PAA Laboratories, Pasching, AT) and $2 \mathrm{mM}$ EDTA (Sigma, Gillingham, UK) per $10^{8}$ cells with $100 \mu \mathrm{l} \mathrm{FCR} \mathrm{blocking} \mathrm{reagent} \mathrm{and} 50 \mu \mathrm{l}$ of microbeads conjugated to monoclonal anti-CD34 antibody (QBEND10) and incubated for $15 \mathrm{~min}$ at $4^{\circ} \mathrm{C}$. The cells were then washed and resuspended in $2 \mathrm{ml}$ of MACS buffer per $10^{8}$ cells. Labeled cells were then passed through a pre-washed MiniMACS column mounted on a magnet. Following this, the column was washed four times with MACS buffer, removed from the magnet and the cells eluted with $2 \mathrm{ml}$ MACS buffer. The purity of the CD34 + fraction was consistently above $96 \%$ as determined by flow cytometry (FACScalibur, Becton Dickinson, Oxford, UK) with anti-CD34 staining. Aliquots were immediately frozen for subsequent lysis for western blot analysis or cultured in leukemic cell growth media for proliferation assays.

Plasmids. pGEX6P1/14-3-3 $\tau$ was generated by subcloning the open-reading frame of human 14-3-3 $\tau$ into pGEX6P1 (Amersham Biosciences, Little Chalfont, UK). pCLAmpho and pcDNA3.1Bcr-Abl were described previously. .6, $^{47}$ PCMV2B_CSDA was generated by subcloning the open-reading frame of human CSDA (Origene, Rockville, MD, USA) into pCMV_Tag2B (Stratagene, La Jolla, CA, USA). pBabe_flagCSDA was cloned by PCR-mediated addition of a $5^{\prime}$ BamHI restriction site and FLAG tag sequence (DYKDDDDK) and $3^{\prime}$ EcoRI restriction site. The CDS with $5^{\prime}$ FLAG tag was then ligated with BamHI- and EcoRI-digested pBabe. Construction of all vectors was confirmed by both restriction digestion and DNA sequencing CSDAS134A point mutant was constructed by site-directed mutagenesis kit (QuickChange $\mathrm{XL}$, Stratagene) according to the manufacturer's instruction using the following primer synthesized by SigmaGenosys (Gillingham, UK): 5'-ACGGAAATATCTGCGCGCTGTAGGA GATGGAGAAA-3'. Point mutation was confirmed by DNA sequencing.

Western blotting and immunoprecipitation. Cells were lysed in icecold buffer containing $1 \%$ (v/v) Triton X-100, 0.05\% (v/v) SDS, $10 \mathrm{mM} \mathrm{NaH}_{2} \mathrm{PO}_{4}$, $150 \mathrm{mM} \mathrm{NaCl}, 5 \mathrm{mM}$ EDTA, $10 \mathrm{mM} \mathrm{NaF}, 1 \mathrm{mM} \mathrm{NaVO}_{3}, 5 \mathrm{nM}$ molybdic acid, $100 \mathrm{nM}$ okadaic acid, $1 \mathrm{mM}$ DTT, $1 \times$ Protease Inhibitor Cocktail (PIC) (Roche, Burgess Hill, UK), $1 \times$ PhosSTOP phosphatase inhibitor cocktail (Roche) and resolved by SDS-PAGE transferred to PVDF membrane (Hybond-P, GE Healthcare, Little Chalfont, UK). Anti-phosphotyrosine antibody (4G10) was a kind gift from Professor B Druker (Oregon Health \& Science University, Portland, OR, USA) and anti-CSDA (ZONAB) antibody was a kind gift from Professor Karl Matter (UCL, London, UK). 
Anti-FLAG (M2) antibody was purchased from Sigma, and anti-c-Abl (Ab3) was purchased from Calbiochem. Anti-PCNA (sc-25280), anti-tubulin (sc-53140), antiRSK1 (sc-231), anti-RSK2 (sc-9986) and anti-YB1 (sc-101198) were purchased from Santa Cruz Biotechnology (Santa Cruz, CA, USA). Anti-phospho-CrkL (3181), anti-phospho-p70S6 kinase (9206) and anti-phosphoYB-1 (2900) were purchased from Cell Signalling Technology (Hitchin, UK). All antibodies were used at 1:1000 dilution. Anti-FLAG immunoprecipitation was performed with M2 agarose beads (Sigma) according to the manufacturer's instructions.

GST-14-3-3-affinity purification. Recombinant GST-14-3-3 was produced from pGEX6P1/14-3-3 $\tau$ transformed in BL21-DE3 bacteria (Stratagene) and purified by binding to glutathione-sepharose beads (GE Healthcare). LAMA84 cells $\left(5 \times 10^{9}\right)$ were left untreated or treated with $2 \mu \mathrm{M} \mathrm{IM}$ and lysed in buffer containing $50 \mathrm{mM}$ Tris-HCl (pH 7.5), $1 \mathrm{mM}$ EDTA, 1 mM EGTA, 1\% (v/v) Triton X-100, $10 \mathrm{mM}$ b-glycerophosphate, $50 \mathrm{mM} \mathrm{NaF}, 1 \mathrm{mM}$ sodium orthovanadate, $5 \mathrm{mM}$ sodium pyrophosphate, $100 \mathrm{nM}$ okadaic acid, $0.27 \mathrm{M}$ sucrose, $1 \mathrm{mM}$ DTT and $1 \times \mathrm{PIC}$. Lysates were clarified and incubated with GST-14-3-3 sepharose beads and 14-3-3 binding proteins eluted with $0.5 \%$ Empigen after stringent washing. Eluates were desalted in Zeba columns (Pierce, Cramlington, UK) and concentrated in YM-10 centrifugal concentrator (Microcon, Watford, UK) and resolved by $4-12 \%$ SDSPAGE (NuPAGE, Invitrogen). Gels were either stained with Gelcode (Pierce) colloidal Coomassie for total protein expression or transferred for western blot analysis with anti-phosphotyrosine antibody (4G10).

Mass spectrometry. Entire sample lanes from Coomassie-stained gels were sectioned into equivalent-sized bands and digested with trypsin. Resultant peptides were subject to LC-MS/MS (Q-TOF, Waters, Elstree, UK) at the Protein Analysis Unit (WHRI, Barts and The London School of Medicine and Dentistry, London, UK: QMUL). Spectra were analyzed using MS/MS Ion Search feature of the MASCOT search engine (Matrix, www.matrixscience.com). Bioinformatic analyses were performed using the Scansite (www.scansite.mit.edu) and Human Protein Reference Database (HPRD, www.hprd.org) on-line software programs.

siRNA screen. Ninety-six-well plates with siRNA targeting sequences (annotated in Supplementary Table 5) to 20 selected candidates (Supplementary Table 4) were custom ordered from Qiagen. A total of $2 \times 10^{4} \mathrm{~K} 562$ cells were transfected with $0.5 \mu \mathrm{l}$ Interferin and $10 \mathrm{nM}$ final siRNA concentration according to the manufacturer's instructions.

Cell proliferation. Proliferation was assessed with MTS reagent (Promega, Madison, WI, USA) according to the manufacturer's instructions. Cells were analyzed at $48 \mathrm{~h}$ for siRNA screen and $72 \mathrm{~h}$ for individual CSDA siRNAs, posttransfection. For inhibitor treatments, $2 \times 10^{4} \mathrm{~K} 562$ or Ramos and $5 \times 10^{4} \mathrm{CD} 34+$ progenitor primary cells were treated 24 and $16 \mathrm{~h}$, respectively, after seeding with inhibitors at indicated concentrations and cultured for $72 \mathrm{~h}$ before MTS reading.

Apoptosis. Cells were analyzed for apoptosis by Annexin V/PI staining as before. ${ }^{48}$ Cells were analyzed at $72 \mathrm{~h}$ post-transfection with control and individual CSDA siRNAs.

Cell cycle. Cells were harvested $72 \mathrm{~h}$ post-transfection with control and individual CSDA siRNAs and fixed in $75 \%$ ice-cold ethanol at $4^{\circ} \mathrm{C}$ for $2 \mathrm{~h}$. Then, cells were stained with PI (Molecular Probes, Eugene, OR, USA) and analyzed by flow cytometry.

Cell transformation. After selection of the transfected stable cell lines, $1 \times 10^{4}$ Rat 1 cells were added to $1.5 \mathrm{ml}$ of growth medium with $0.35 \%$ agar and layered onto $2 \mathrm{ml}$ of $0.5 \%$ agar base in six-well plates. Cells were fed with $2 \mathrm{ml}$ of medium every 3 days for 4 weeks, after which colonies were stained with MTT $(0.5 \mathrm{mg} / \mathrm{ml})$ for $30 \mathrm{~min}$ and counted. Colonies visible under a microscope were counted as positive for growth.

Statistics. We performed statistical analysis using ANOVA. Results were considered significant at $P<0.05$.

\section{Conflict of interest}

The authors declare no conflict of interest.
Acknowledgements. This work was supported by Cancer Research UK and Leuka. Author contributions: DS designed and performed experiments and helped write manuscript; PL, MY, GN and YKSM performed experiments; JVM co-conceived, designed and supervised initial project execution and helped write manuscript; and SB co-conceived, designed and supervised execution of project and wrote final manuscript.

1. Melo JV, Barnes DJ. Chronic myeloid leukaemia as a model of disease evolution in human cancer. Nat Rev Cancer 2007; 7: 441-453.

2. Gordon MY, Dowding CR, Riley GP, Goldman JM, Greaves MF. Altered adhesive interactions with marrow stroma of haematopoietic progenitor cells in chronic myeloid leukaemia. Nature 1987; 328: 342-344

3. Verfaillie CM, Hurley R, Lundell $\mathrm{BI}$, Zhao $\mathrm{C}$, Bhatia R. Integrin-mediated regulation of hematopoiesis: do BCR/ABL-induced defects in integrin function underlie the abnormal circulation and proliferation of CML progenitors? Acta Haematol 1997; 97: 40-52.

4. Bedi A, Zehnbauer BA, Barber JP, Sharkis SJ, Jones RJ. Inhibition of apoptosis by BCRABL in chronic myeloid leukemia. Blood 1994; 83: 2038-2044.

5. Goldman JM, Melo JV. BCR-ABL in chronic myelogenous leukemia-how does it work? Acta Haematol 2008; 119: 212-217.

6. Druker BJ, Sawyers CL, Kantarjian H, Resta DJ, Reese SF, Ford JM et al. Activity of a specific inhibitor of the BCR-ABL tyrosine kinase in the blast crisis of chronic myeloid leukemia and acute lymphoblastic leukemia with the Philadelphia chromosome. $N$ Engl J Med 2001; 344: 1038-1042.

7. Sawyers CL, Hochhaus A, Feldman E, Goldman JM, Miller CB, Ottmann OG et al. Imatinib induces hematologic and cytogenetic responses in patients with chronic myelogenous leukemia in myeloid blast crisis: results of a phase II study. Blood 2002; 99: 3530-3539.

8. Basu S, Totty NF, Irwin MS, Sudol M, Downward J. Akt phosphorylates the Yes-associated protein, YAP, to induce interaction with 14-3-3 and attenuation of p73-mediated apoptosis. Mol Cell 2003; 11: 11-23.

9. Skorski T, Bellacosa A, Nieborowska-Skorska M, Majewski M, Martinez R, Choi JK et al Transformation of hematopoietic cells by $\mathrm{BCR} / \mathrm{ABL}$ requires activation of a $\mathrm{PI}-3 \mathrm{k} / \mathrm{Akt}$ dependent pathway. EMBO J 1997; 16: 6151-6161.

10. Skorski T, Kanakaraj P, Nieborowska-Skorska M, Ratajczak MZ, Wen SC, Zon G et al Phosphatidylinositol-3 kinase activity is regulated by $B C R / A B L$ and is required for the growth of Philadelphia chromosome-positive cells. Blood 1995; 86: 726-736.

11. Morrison DK. The 14-3-3 proteins: integrators of diverse signaling cues that impact cell fate and cancer development. Trends Cell Biol 2009; 19: 16-23.

12. Hermeking H. The 14-3-3 cancer connection. Nat Rev Cancer 2003; 3: 931-943.

13. Sano H, Liu SC, Lane WS, Piletz JE, Lienhard GE. Insulin receptor substrate 4 associates with the protein IRAS. J Biol Chem 2002; 277: 19439-19447.

14. Aitken A, Baxter H, Dubois T, Clokie S, Mackie S, Mitchell K et al. Specificity of 14-3-3 isoform dimer interactions and phosphorylation. Biochem Soc Trans 2002; 30: 351-360.

15. Benzinger A, Muster N, Koch HB, Yates III JR, Hermeking H. Targeted proteomic analysis of 14-3-3 sigma, a p53 effector commonly silenced in cancer. Mol Cell Proteomics 2005; 4: 785-795.

16. Ewing RM, Chu $\mathrm{P}$, Elisma F, Li H, Taylor $\mathrm{P}$, Climie $\mathrm{S}$ et al. Large-scale mapping of human protein-protein interactions by mass spectrometry. Mol Syst Biol 2007; 3: 89.

17. Jin J, Smith FD, Stark C, Wells CD, Fawcett JP, Kulkarni S et al. Proteomic, functional, and domain-based analysis of in vivo 14-3-3 binding proteins involved in cytoskeletal regulation and cellular organization. Curr Biol 2004; 14: 1436-1450.

18. Nellist M, Goedbloed MA, de Winter C, Verhaaf B, Jankie A, Reuser AJ et al. Identification and characterization of the interaction between tuberin and 14-3-3zeta. J Biol Chem 2002; 277: 39417-39424.

19. Pozuelo Rubio M, Peggie M, Wong BH, Morrice N, MacKintosh C. 14-3-3s regulate fructose-2,6-bisphosphate levels by binding to PKB-phosphorylated cardiac fructose-2,6bisphosphate kinase/phosphatase. EMBO J 2003; 22: 3514-3523.

20. Trinczek B, Brajenovic M, Ebneth A, Drewes G. MARK4 is a novel microtubule-associated proteins/microtubule affinity-regulating kinase that binds to the cellular microtubule network and to centrosomes. J Biol Chem 2004; 279: 5915-5923.

21. Tzivion G, Luo ZJ, Avruch J. Calyculin A-induced vimentin phosphorylation sequesters 14-3-3 and displaces other 14-3-3 partners in vivo. J Biol Chem 2000; 275: 29772-29778.

22. Yoshida K, Yamaguchi T, Natsume T, Kufe D, Miki Y. JNK phosphorylation of 14-3-3 proteins regulates nuclear targeting of c-Abl in the apoptotic response to DNA damage. Nat Cell Biol 2005; 7: 278-285.

23. Pozuelo Rubio M, Geraghty KM, Wong BH, Wood NT, Campbell DG, Morrice N et al. 14-33-affinity purification of over 200 human phosphoproteins reveals new links to regulation of cellular metabolism, proliferation and trafficking. Biochem J 2004; 379 (Part 2): 395-408.

24. Sourisseau T, Georgiadis A, Tsapara A, Ali RR, Pestell R, Matter $\mathrm{K}$ et al. Regulation of PCNA and cyclin D1 expression and epithelial morphogenesis by the ZO-1-regulated transcription factor ZONAB/DbpA. Mol Cell Biol 2006; 26: 2387-2398.

25. Balda MS, Garrett MD, Matter K. The ZO-1-associated Y-box factor ZONAB regulates epithelial cell proliferation and cell density. J Cell Biol 2003; 160: 423-432.

26. Wang GR, Zheng Y, Che XM, Wang XY, Zhao JH, Wu KJ et al. Upregulation of human DNA binding protein A (dbpA) in gastric cancer cells. Acta Pharmacol Sin 2009; 30: 1436-1442. 
27. Crawford NP, Yang $\mathrm{H}$, Mattaini $\mathrm{KR}$, Hunter $\mathrm{KW}$. The metastasis efficiency modifier ribosomal RNA processing 1 homolog $B(R R P 1 B)$ is a chromatin-associated factor. $J$ Biol Chem 2009; 284: 28660-28673.

28. Sutherland BW, Kucab J, Wu J, Lee C, Cheang MC, Yorida E et al. Akt phosphorylates the Y-box binding protein 1 at Ser102 located in the cold shock domain and affects the anchorage-independent growth of breast cancer cells. Oncogene 2005; 24: 4281-4292.

29. Feller SM. Crk family adaptors-signalling complex formation and biological roles Oncogene 2001; 20: 6348-6371.

30. Sattler M, Salgia R, Okuda K, Uemura N, Durstin MA, Pisick E et al. The proto-oncogene product $\mathrm{p} 120 \mathrm{CBL}$ and the adaptor proteins $\mathrm{CRKL}$ and c-CRK link c-ABL, p190BCR/AB and $\mathrm{p} 210 \mathrm{BCR} / \mathrm{ABL}$ to the phosphatidylinositol-3' kinase pathway. Oncogene 1996; 12 839-846.

31. Roux PP, Richards SA, Blenis J. Phosphorylation of p90 ribosomal S6 kinase (RSK) regulates extracellular signal-regulated kinase docking and RSK activity. Mol Cell Biol 2003; 23: 4796-4804.

32. Richards SA, Dreisbach VC, Murphy LO, Blenis J. Characterization of regulatory events associated with membrane targeting of p90 ribosomal S6 kinase 1. Mol Cell Biol 2001; 21: $7470-7480$

33. Kin Y, Li G, Shibuya M, Maru Y. The Dbl homology domain of BCR is not a simple spacer in P210BCR-ABL of the Philadelphia chromosome. J Biol Chem 2001; 276: 39462-39468.

34. Pendergast AM, Quilliam LA, Cripe LD, Bassing CH, Dai Z, Li N et al. BCR-ABL-induced oncogenesis is mediated by direct interaction with the $\mathrm{SH} 2$ domain of the GRB-2 adaptor protein. Cell 1993; 75: 175-185.

35. Anjum R, Blenis J. The RSK family of kinases: emerging roles in cellular signalling. Nat Rev Mol Cell Biol 2008; 9: 747-758.

36. Roux PP, Shahbazian D, Vu H, Holz MK, Cohen MS, Taunton J et al. RAS/ERK signaling promotes site-specific ribosomal protein S6 phosphorylation via RSK and stimulates capdependent translation. J Biol Chem 2007; 282: 14056-14064.

37. Lima WR, Parreira KS, Devuyst O, Caplanusi A, N'Kuli F, Marien B et al. ZONAB promotes proliferation and represses differentiation of proximal tubule epithelial cells. $J$ Am Soc Nephrol 2010; 21: 478-488.

38. Bader AG, Vogt PK. Phosphorylation by Akt disables the anti-oncogenic activity of YB-1. Oncogene 2008; 27: 1179-1182.
39. Evdokimova V, Ruzanov P, Anglesio MS, Sorokin AV, Ovchinnikov LP, Buckley J et al. Aktmediated YB-1 phosphorylation activates translation of silent mRNA species. Mol Cell Biol 2006; 26: 277-292.

40. Evdokimova V, Tognon C, Ng T, Ruzanov P, Melnyk N, Fink D et al. Translational activation of snail 1 and other developmentally regulated transcription factors by YB-1 promotes an epithelial-mesenchymal transition. Cancer Cell 2009; 15: 402-415.

41. Ma Z, Cools J, Marynen P, Cui X, Siebert R, Gesk S et al. Inv(2)(p23q35) in anaplastic large-cell lymphoma induces constitutive anaplastic lymphoma kinase (ALK) tyrosine kinase activation by fusion to ATIC, an enzyme involved in purine nucleotide biosynthesis. Blood 2000; 95: 2144-2149.

42. Kanno H, Fujii H, Hirono A, Ishida $Y$, Ohga S, Fukumoto $Y$ et al. Molecular analysis of glucose phosphate isomerase deficiency associated with hereditary hemolytic anemia. Blood 1996; 88: 2321-2325.

43. Scatena R, Bottoni P, Pontoglio A, Mastrototaro L, Giardina B. Glycolytic enzyme inhibitors in cancer treatment. Expert Opin Invest Drugs 2008; 17: 1533-1545.

44. Sheng H, Niu B, Sun H. Metabolic targeting of cancers: from molecular mechanisms to therapeutic strategies. Curr Med Chem 2009; 16: 1561-1587.

45. Yoshida C, Yoshida F, Sears DE, Hart SM, Ikebe D, Muto A et al. Bcr-Abl signaling through the PI-3/S6 kinase pathway inhibits nuclear translocation of the transcription factor Bach2, which represses the antiapoptotic factor heme oxygenase-1. Blood 2007; 109: 1211-1219.

46. Griswold IJ, MacPartlin M, Bumm T, Goss VL, O'Hare T, Lee KA et al. Kinase domain mutants of Bcr-Abl exhibit altered transformation potency, kinase activity, and substrate utilization, irrespective of sensitivity to imatinib. Mol Cell Biol 2006; 26: 6082-6093.

47. Naviaux RK, Costanzi E, Haas M, Verma IM. The PCL vector system: rapid production of helper-free, high-titer, recombinant retroviruses. J Virol 1996; 70: 5701-5705.

48. Tomlinson V, Gudmundsdottir K, Luong P, Leung KY, Knebel A, Basu S. JNK phosphorylates Yes-associated protein (YAP) to regulate apoptosis. Cell Death Dis 2010; 1: e29.

Cell Death and Disease is an open-access journal published by Nature Publishing Group. This work is licensed under the Creative Commons Attribution-Noncommercial-No Derivative Works 3.0 Unported License. To view a copy of this license, visit http://creativecommons.org/licenses/by-nc-nd/3.0/

\section{Supplementary Information accompanies the paper on Cell Death and Disease website (http://www.nature.com/cddis)}

